\title{
Fat Quantification in MRI-defined lumbar muscles
}

\author{
Joseph Antony*, Kevin McGuinness*, Neil Welch ${ }^{\dagger}$, Joe Coyle ${ }^{\dagger}$, Andy Franklyn-Miller ${ }^{\dagger}$, \\ Noel E OConnor*, Kieran Moran ${ }^{\ddagger}$ \\ * Insight Centre for Data Analytics, Dublin City University, Dublin, Ireland. \\ $\dagger$ Sports Medicine Department, Sports Surgery Clinic, Dublin, Ireland. \\ $\ddagger$ School of Health and Human Performance, Dublin City University, Dublin, Ireland. \\ e-mail: joseph.antony2@mail.dcu.ie
}

\begin{abstract}
Some studies suggest fat infiltration in the lumbar muscles (LM) is associated with lower back pain (LBP) in adults. Usually fat in MRI-defined lumbar muscles is qualitatively evaluated by visual grading via a 3 point scale, whereas a quantitative continuous $(0-100 \%)$ approach may provide a greater insight. In this paper, we propose a method to precisely quantify the fat content / infiltration in a user-defined region of the lumbar muscles, which may aid better diagnosis. The key steps are segmenting the region of interest (ROI) from the lumbar muscles, identifying the fatty regions in the segmented region based on the selected threshold and softness levels, computing the parameters (such as total and region-wise fat content percentage, total-cross sectional area (TCSA), functional cross-sectional area (FCSA)) and exporting the computations and associated patient information from the MRI, into a database. A standalone application using MATLAB R2010a was developed to perform the required computations along with an intuitive GUI.
\end{abstract}

Index Terms-fat infiltration, lumbar muscles, region of interest, region-wise segmentation, fat percentage, graphical user interface.

\section{INTRODUCTION}

Several MRI studies have related lower back pain in adults to fat infiltration in the lumbar muscles [1], [3], [7], [9], [12], [14], [16]. However, others suggest there is no relationship [8]. A challenge to greater understanding is the lack of precision in quantifying fat infiltration. Generally fat depositions in the lumbar muscles are estimated qualitatively [3], [9], [10] using a visual grading system (e.g. $0,1,2)$ [1], but these overlook small changes in muscle composition [8] and do not provide precision of measurement.

We have adopted a quantitative approach to precisely quantify the amount of fat deposition in the lumbar muscles. The proposed method of quantifying fat infiltration in the lumbar muscles is proposed as a supporting system to the physicians to make better diagnosis as well as to check the effectiveness of the exercises or workouts being prescribed [13], [15] in rehabilitation programs [8]. In addition we quantify the fat content in the erector spinae muscles in a region wise manner with respect to the centre of the spinal column [17], [18], [19], which represents the axis of spinal rotation [17]. From a bio-mechanical perspective of lower back pain, damage to the muscle region further from the axis of spinal rotation may have greater effect on the pain levels [18], [19], because the moment of force produced by the muscle is dependent not only on the amount of muscle or muscle force, but also the distribution of muscle relative to the axis of rotation (the moment of force $\tau=\|r\| .\|F\| . \sin (\theta)$, where $r$ is the (lever arm) displacement vector, $F$ is the force vector, $\theta$ is the angle between lever arm and force vector).

There are four key steps in this process. The first step is defining the region of interest (ROI) in the MRI-defined lumbar muscles and then segmenting the ROI [2], [3]. The second step is detecting the fatty regions based on the threshold [4], [5] and softness level selected by the user, and computing the fat content percentage [2], [4], [5]. The third step is subdividing the ROI into smaller segments with reference to the centre of the spinal column. The final step is computing the total cross-sectional area [2], [3], the functional cross-sectional area [2], [3], [6] and the fat content percentage in every region. A stand-alone graphical user interface (GUI) using Matlab R2010a was developed based on the four steps, with interactive controls for selecting the region of interest (ROI) from the input image, threshold adjustment, softness level adjustment, displaying the intermediate results and appending the computed results into an existing database.

The main contributions of this work are quantifying the fat content based on a region wise segmentation of the lumbar muscles with reference to the center of the spinal column and development of the standalone application with intuitive graphical user interface (GUI). The main difference in our work with respect to previous work reported [1], [2], [3], [8], [9], [10] is the use of a sigmoid function for quantifying the fat content in the lumbar muscles, which provides an additional sharpness control along with the threshold for identifying the fatty regions in the lumbar muscles.

\section{RELATED WORK}

Qualitative [1], [3], [7], [9], [12], [14], [16] as well as quantitative approaches [8], [11] are used for the study of fat deposition in muscles. In the qualitative approach [1], [9], the fat infiltration in the lumbar multifidus muscles is visually graded, using the standard scale of 0,1 and 2 [1], [9]. In the grading system as shown in Figure 1, grade 0 attributes to "normal" or "none" representing 0-10\% fat within the muscles, grade 1 attributes to "slight" or "mild" for 10-50\% fat and grade 2 attributes to "severe" for more than $50 \%$ fat. This grading system has been adapted from previous studies 
involving low-field MRI [1], [15], [16] and showed good intraobserver and good inter-observer agreement. Similarly, in an another grading system [8], the fat content in the supraspinatus muscles are graded in the scale of $0-4$.

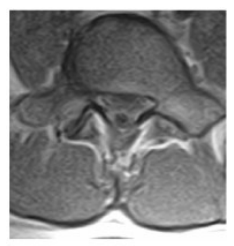

Grade 0 (none)

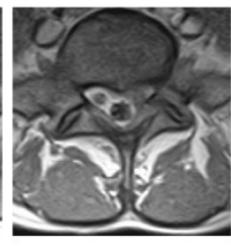

Grade 1 (slight)

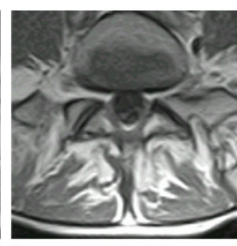

Grade 2 (severe)
Fig. 1. Visual grading for fat infiltration in the lumbar multifidus muscle.

According to the study involving subjects with sway-back posture [2], fat infiltration in the lumbar multifidus and the lumbar erector spinae muscles are related to the muscle atrophy [9] and consequently back pain. One of the main reasons considered for the relationship between fat infiltration in the lumbar muscles and back pain is the increased intramuscular fat deposits that may affect the contractility of the muscles required for the control of spinal orientation and inter-vertebral motion [1], [2], [8], [9].

\section{MATERIALS AND MethodS}

\section{A. Experimental data}

The image acquisition was performed in the Sports Surgery Clinic, Santry Demense, Santry, Dublin. Images were acquired on a General Electric (GE) Signa HDxt 3 tesla scanner using an 8 channel phased array spine coil. Axial T2 FRFSE (Fast Recovery Fast Spin Echo) sequences were acquired on patients under investigation for low back pain. Imaging parameters included; 4000/108 Repetition time/Time to Echo (TR/TE), $320 \times 244$ matrix, $20 \times 20 \mathrm{~cm}$ field of view, slice thickness $4 \mathrm{~mm}$ with a $1 \mathrm{~mm}$ gap. MR Lumbar spine images of two patients under study of low back pain and fat infiltration in lumbar spine muscles were considered for the analysis. Using a DICOM converter, the lumbar spine MR images were converted to PNG format for analysing in MATLAB.

\section{B. Segmenting the region of interest}

The first step is selecting the region of interest (ROI) from the MRI-defined lumbar muscles, which can be any among the erector spinae (ES) muscles, lumbar multifidus muscles (LMM) or psaos muscles, located either on the right or the left side of the central spinal column [2],[3]. The user has to define the ROI by drawing a freehand mask over the input image as shown in Figure 2. The mask is realized as a set of points in 2D coordinates, $f(x, y)$. Then using the set of points $f(x, y)$, the region inside the mask is cropped from the input image.

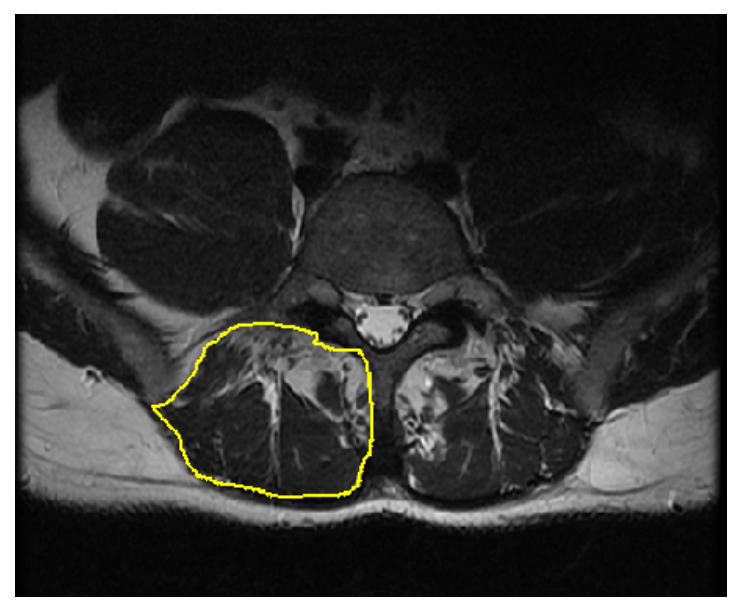

Fig. 2. MRI input image with user defined mask.

\section{Identifying the fatty regions}

The pixel signal intensity (SI) variations between the muscle and the fatty region can be used to distinguish the fatty region from muscle region [2],[3],[6],[9],[11]. By using an appropriate threshold, the pixels in the fatty regions of the segmented lumbar muscles are detected [4],[5]. Instead of using a hard threshold, the sigmoid function is proposed in this paper for setting the threshold which aids in terms of an additional softness level control for detecting the fatty regions. The sigmoid function refers to a special case of a logistic function defined by the equation:

$$
s(x, a, c)=\frac{1}{1+e^{-a(x-c)}}
$$

where $c$ is the centre and $a$ is the slope control. Every pixel $p(x, y)$ in the segmented lumbar muscle region is subjected to the sigmoid function $s(x, c, a)$ which gives a clear discrimination between the muscle region and the fatty region $p_{f a t}(x, y)$ as shown in Fig. 5.

$$
p_{\text {fat }}(x, y)= \begin{cases}1, & \text { if } p(x, y) \in \text { "fat" } \\ 0, & \text { otherwise }\end{cases}
$$
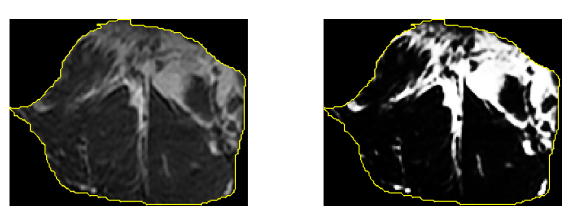

Fig. 3. Detected fatty region from the segmented muscle region (with threshold $=80$ and softness $=0.1$ )

In the sigmoid function, choosing different values for the centre $c$ is associated to the threshold selection for discerning the fatty region from the muscle region. Similarly varying the values of slope control $a$ in the sigmoid function is associated to the softness level of the discerned fatty region edges. Pixel $p(x, y)$ belongs to "fat", if the pixel intensity falls above 
the threshold selected by adjusting the centre $c$ value in the sigmoid function $s(x, c, a)$.

By taking the ratio between the number of pixels $(N)$ in the segmented lumbar muscle region to the total pixels in the detected fatty region $\sum p_{f a t}(x, y)$, the total fat content is calculated.

$$
\text { Total fat content } \%=\left(\frac{\sum p_{f a t}(x, y)}{N}\right) \times 100
$$

For example, considering the segmented region shown in Figure 3, the total pixels in the segmented lumbar muscle region were 21,156 and the pixels in the fatty region were 3733 and the computed total fat content was $17.6 \%$.

\section{Region wise quantification}

The next step is to quantify the fat content in various regions of the segmented muscles. The segmented muscles could be sub-divided into many regions. We have subdivided the segmented muscles into six regions with reference to the centre of spinal column, as shown in Figure 5 or Figure 6 . The use of six segments was based solely on visual observation and clearly needs further research. Generally, the segmented muscle region is irregularly shaped. The boundary points are extracted and used to sub-divide the region into smaller segments. After obtaining the various segments, the fat content percentage in each segment is calculated.

The following steps are used to subdivide the segmented region and to perform the computations:

Step 1: The centre of the spinal column $c(x, y)$ is indicated by the user.

Step 2: A radial line from the centre of the spinal column $c(x, y)$, which passes through the centroid of the segmented muscle region and that bisects the erector spinae muscles is plotted as shown in Figure 4.

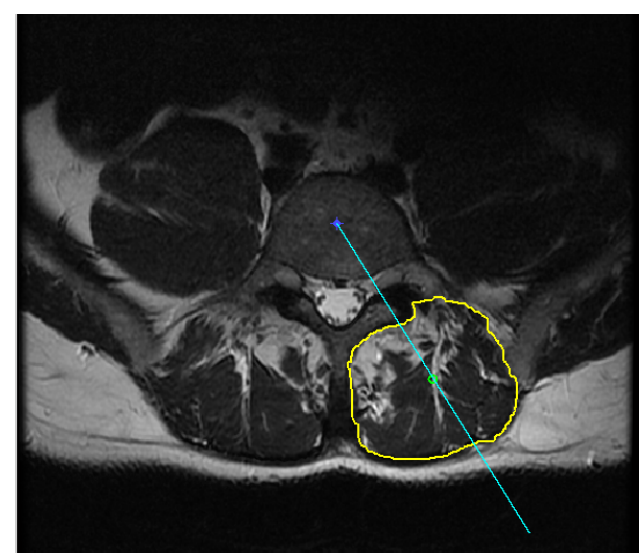

Fig. 4. Input image with the radial line from the centre of spinal column (Right Erector Spinae Muscles).

Step 3: Considering the radial line as vector $v 1$ and a horizontal line through the centre of the spinal column $c(x, y)$ as vector $v 2$, the angle $(\theta)$ between the vectors $v 1$ and $v 2$ is calculated.
Step 4: The angle $(\theta)$ is used to identify, whether the segmented muscle region lies either on the right side or the left side of the spinal column. If the angle $(\theta)$ is less than $90^{\circ}$ the segmented muscle region is considered to be on the right side and it is rotated by angle $(\theta)$ in the counter-clockwise direction as shown in Figure 5, else the segmented muscle region is considered to be on the left side and it is rotated by an angle $(180-\theta)$ in the clockwise direction as shown in Figure 6.
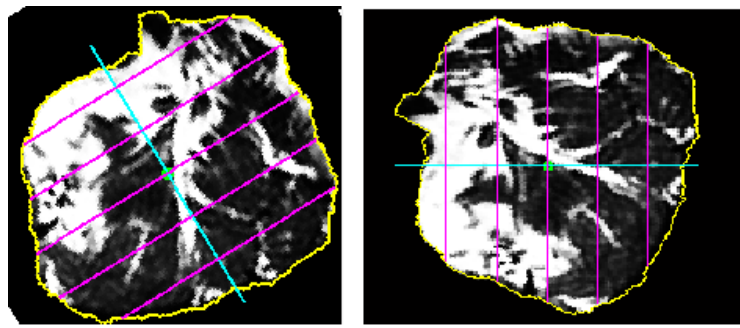

Fig. 5. Segmented Right Erector Spinae muscle rotated by angle $(\theta)$ in counter-clockwise direction.
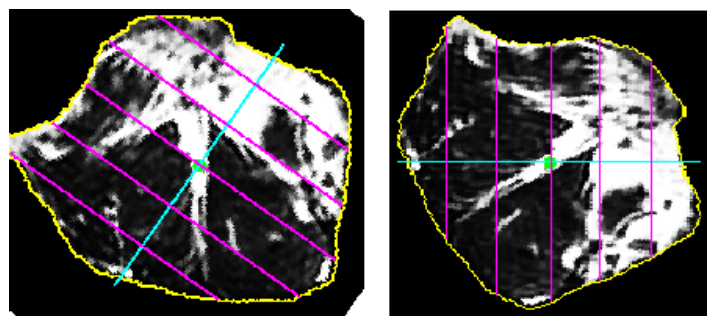

Fig. 6. Segmented Left Erector Spinae muscle rotated by angle $(180-\theta)$ in clockwise direction.

Step 5: From the segmented muscle region, the contour as a set of points, $f(x, y)$ is extracted. Then the vectors $[X]$ and $[Y]$ pertaining to the contour points of the $\mathrm{X}$-coordinates and Y-Coordinates, respectively, were extracted.

Step 6: The maximum and minimum values of $[X]$ and $[Y]$ are found, which gives the extremities of the irregular shaped segmented muscle region.

Step 7: The length $(L)$ of the segmented region, which is the difference between the maxima and minima of $[X]$ is calculated. Further, the length $(L)$ is used to sub-divide the segmented muscle region.

Step 8: To have six sub-divisions, five equidistant vertical lines are plotted over the segmented lumbar muscle region at regular intervals $(L / 6)$ from minima of $[X]$. These vertical lines are plotted from minima of $[Y]$ to maxima of $[Y]$ so that every line touches the contours of the segmented muscle region as shown in Figure 5 and Figure 6.

Step 9: Considering all seven hyperplanes, one each at the minima and maxima of $[X]$ and one at each of the five vertical lines, the fat content in six smaller segments are calculated.

Step 10: The fat content in every smaller segment is calculated by subjecting every pixel inside the region to the 
sigmoid function with the pre-selected threshold and softness level.

\section{E. GUI and computations}

A standalone Graphical User Interface (GUI) using Matlab $\mathrm{R} 2010 \mathrm{a}$ was developed with the required interactive controls. Initially the GUI, allows the user to select an input image. Then the user can define the region of interest (ROI), which is a freehand mask drawn over the input image. The 'Brightness' sliding control allows the user to adjust the brightness of the input image. Once the user draws the freehand mask, the ROI is segmented and displayed in the GUI. By default the threshold and softness values are set at 60 and 0.1 respectively (based on experience). Initially with these values, the fatty region are identified from the segmented lumbar muscle and displayed in the GUI. Based on visual inspection, suitable values for threshold and softness can be selected by adjusting the 'Threshold' and 'Softness' sliding controls respectively.

The total fat content percentage, total cross-sectional area (TCSA) and functional cross-sectional area (FCSA) in $\mathrm{mm}^{2}$ can be calculated at any stage by pressing the 'Compute' button. The computation of fat content percentage, TCSA, and FCSA are performed following the previous studies [2],[3], [8],[6]. The physical pixel size (psize) required for the calculation of TCSA and FCSA is read from MRI meta-data. The computations performed are:

$$
\begin{gathered}
\text { TCSA }=(N \times p \text { size }) \text { in } \mathrm{mm}^{2} \\
\text { FCSA }=\left(\left(N-\sum p_{f a t}(x, y)\right) \times p \text { size }\right) \text { in } m m^{2}
\end{gathered}
$$

where $N$ is the number of pixels in the segmented region, $\sum p_{f a t}(x, y)$ is the total pixels in the fatty region. Total fat content percentage is calculated as per equation (3).

By using the drop down menu 'Label Region' the user indicates the region of interest. The list of regions included in the menu are Right Erector Spinae muscles, Left Erector Spinae muscles, Left Lumbar Multifidus Muscles, Right Lumbar Multifidus Muscles, Right Psoas Muscles, Left Psoas Muscles.

The region wise quantification of fat content in the Erector Spinae (ES) muscles, either on left or right side of the spinal column, is carried out by selecting 'Segment'. The fat content percentage in the six sub-divisions of the segmented regions, labelled R1 to R6 from top to bottom respectively, are computed and displayed.

The GUI was iteratively developed based on feedback from experts. The softness control, computation of total and functional cross-sectional area, region-wise segmentation with reference to the centre of spinal column were included, based on expert suggestions.

\section{REsults}

For the input images with the defined region of interest (ROI) being the lumbar multifidus muscles (LMM) (shown in Figure 7 and Figure 8): fat content, total cross sectional area (TCSA) and functional cross sectional area (FCSA) were calculated with threshold 70 and softness level 0.1 , shown in Table I and II. Interestingly, both images would be classified as a scale 1 fat infiltration, even though they clearly differ (by $11.3 \%)$.

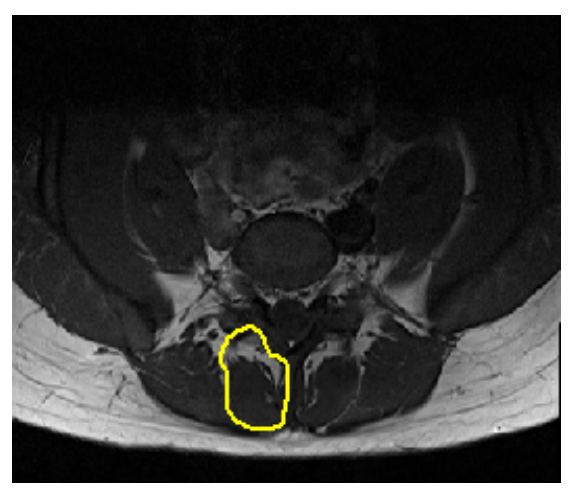

Fig. 7. Input image with ROI as LMM (left)

TABLE I

FAT QUANTIFICATION: Lumbar MUlTifidus Muscles (LEFT)

\begin{tabular}{|c|c|c|c|}
\hline Segmented ROI & Fat Content & TCSA & FCSA \\
\hline
\end{tabular}

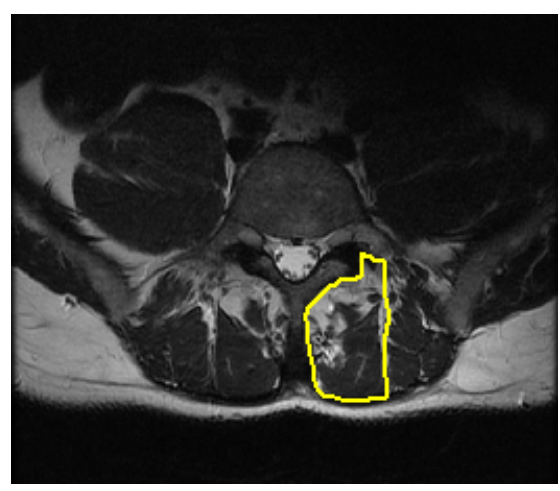

Fig. 8. Input image with ROI as LMM (Right)

TABLE II

FAT QuANTIFICATION: Lumbar Multifidus Muscles (RIGHT)

\begin{tabular}{|l|l|l|l|}
\hline Segmented ROI & Fat Content & TCSA & FCSA \\
\hline
\end{tabular}


From the MRI images of two different patients, the selected region of interest (ROI) being erector spinae Muscles (ES) either on left or right side of the spinal column the parameters (such as region wise fat content, total fat content, total cross sectional area (TCSA) and functional cross sectional area (FCSA)) were calculated. The results for Patient I and Patient II are shown in table III and IV, respectively.

TABLE III

PATIENT I: FAT QUANTIFICATION RESULTS

\begin{tabular}{|c|c|}
\hline Segmented Region of Interest & Computations \\
\hline$\rightarrow 2$ & $\begin{array}{l}\text { Region-wise Fat } \\
\text { R1: } 5.1 \% \text { (Top) } \\
\text { R2: } 3.8 \% \\
\text { R3: } 1.4 \% \\
\text { R4: } 0.9 \% \\
\text { R5: } 0.6 \% \\
\text { R6: } 0.4 \% \\
\text { Total Fat : } 12.1 \% \\
\text { TCSA : } 44 \mathrm{~mm}^{2} \\
\text { FCSA : } 39 \mathrm{~mm}^{2}\end{array}$ \\
\hline & $\begin{array}{l}\text { Region-wise Fat } \\
\text { R1: } 3.5 \% \text { (Top) } \\
\text { R2: } 5.1 \% \\
\text { R3: } 2.9 \% \\
\text { R4: } 1.8 \% \\
\text { R5: } 0.8 \% \\
\text { R6: } 0.4 \% \\
\text { Total Fat : } 14.4 \% \\
\text { TCSA : } 45 \mathrm{~mm}^{2} \\
\text { FCSA : } 38 \mathrm{~mm}^{2}\end{array}$ \\
\hline & $\begin{array}{l}\text { Region-wise Fat } \\
\text { R1: } 1.7 \% \text { (Top) } \\
\text { R2: } 7.4 \% \\
\text { R3: } 3.5 \% \\
\text { R4: } 2.1 \% \\
\text { R5: } 1.1 \% \\
\text { R6: } 0.6 \% \\
\text { Total Fat : } 15.5 \% \\
\text { TCSA : } 40 \mathrm{~mm}^{2} \\
\text { FCSA : } 34 \mathrm{~mm}^{2}\end{array}$ \\
\hline & $\begin{array}{l}\text { Region-wise Fat } \\
\text { R1: } 3.1 \% \text { (Top) } \\
\text { R2: } 5.6 \% \\
\text { R3: } 3.1 \% \\
\text { R4: } 1.2 \% \\
\text { R5: } 0.3 \% \\
\text { R6: } 0.2 \% \\
\text { Total Fat : } 13.2 \% \\
\text { TCSA : } 21 \mathrm{~mm}^{2} \\
\text { FCSA : } 18 \mathrm{~mm}^{2}\end{array}$ \\
\hline
\end{tabular}

The screen shots of the GUI from the quantification of fat content in erector spinae muscles (left and right) are shown in Figure 9 and Figure 10, respectively.

\section{CONCLUSION AND FUtURE WORK}

We have proposed a method to quantify the cross-sectional area and distribution of fat in the MRI scans of lumbar
TABLE IV

PATIENT II: FAT QUANTIFICATION RESULTS

\begin{tabular}{|c|c|}
\hline Segmented Region of Interest & Computations \\
\hline & $\begin{array}{l}\text { Region-wise Fat } \\
\text { R1: } 1.3 \% \text { (Top) } \\
\text { R2: } 5.9 \% \\
\text { R3: } 4.7 \% \\
\text { R4: } 2.2 \% \\
\text { R5: } 1.0 \% \\
\text { R6: } 0.6 \% \\
\text { Total Fat : } 14.8 \% \\
\text { TCSA : } 81 \mathrm{~mm}^{2} \\
\text { FCSA : } 69 \mathrm{~mm}^{2}\end{array}$ \\
\hline & $\begin{array}{l}\text { Region-wise Fat } \\
\text { R1: } 5.7 \% \text { (Top) } \\
\text { R2: } 9.3 \% \\
\text { R3: } 7.2 \% \\
\text { R4: } 5.1 \% \\
\text { R5: } 3.4 \% \\
\text { R6: } 1.7 \% \\
\text { Total Fat : } 29.6 \% \\
\text { TCSA : } 79 \mathrm{~mm}^{2} \\
\text { FCSA : } 56 \mathrm{~mm}^{2}\end{array}$ \\
\hline & $\begin{array}{l}\text { Region-wise Fat } \\
\text { R1: } 3.4 \% \text { (Top) } \\
\text { R2: } 9.9 \% \\
\text { R3: } 9.9 \% \\
\text { R4: } 5.5 \% \\
\text { R5: } 2.5 \% \\
\text { R6: } 0.9 \% \\
\text { Total Fat : } 29.8 \% \\
\text { TCSA : } 83 \mathrm{~mm}^{2} \\
\text { FCSA : } 58 \mathrm{~mm}^{2}\end{array}$ \\
\hline & $\begin{array}{l}\text { Region-wise Fat } \\
\text { R1: } 6.2 \% \text { (Top) } \\
\text { R2: } 8.7 \% \\
\text { R3: } 10.0 \% \\
\text { R4: } 6.9 \% \\
\text { R5: } 6.0 \% \\
\text { R6: } 1.5 \% \\
\text { Total Fat : } 36.4 \% \\
\text { TCSA : } 52 \mathrm{~mm}^{2} \\
\text { FCSA : } 33 \mathrm{~mm}^{2}\end{array}$ \\
\hline
\end{tabular}

muscles. We clearly show its advantage in precisely quantifying these measures over the commonly employed method of subjective evaluation (e.g. scale $0,1,2$ ). This method will help future studies more accurately examine the relationship between fat infiltration and lower back pain; and if a relationship is apparent, it may provide a greater insight into the rehabilitation process beyond reliance on a patient's reporting of pain.

We also have implemented a method to quantify the fat content in a region wise manner with reference to the spinal column. This may be important as the distribution of fat and muscle relative to the axis of rotation produced by the whole muscle has functional implications for both the control of the spine and loading on the muscles. In addition, we have 
developed a graphical user interface (GUI) with interactive controls to perform the required computations, which can act as a supporting system to physicians.

Future work should focus on techniques such as 'active contour model' or 'live wire on the fly', which could avoid manual errors in precisely defining the ROI. In the GUI, centre of the spinal column is read as a user-input, instead the centre could be automatically detected by manually annotating the cohort of MRI images and using 'geometric feature learning' algorithms. Automating the system further is a challenge because the signal intensity of tissues within an MRI varies from scan to scan (even on the same person). It may be useful to explore the use of the signal intensity of the spinal column itself as a means of standardising the selection of the intensity threshold to distinguish between fat and muscle.

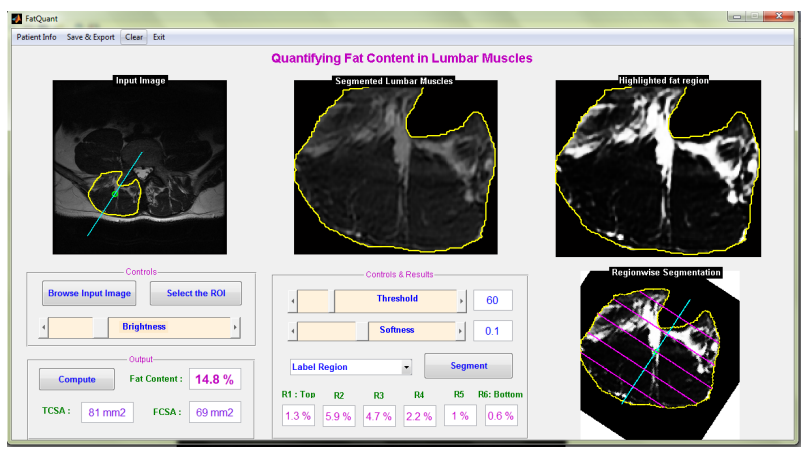

Fig. 9. GUI screen shot: Quantifying fat content in ES Muscles (Left)

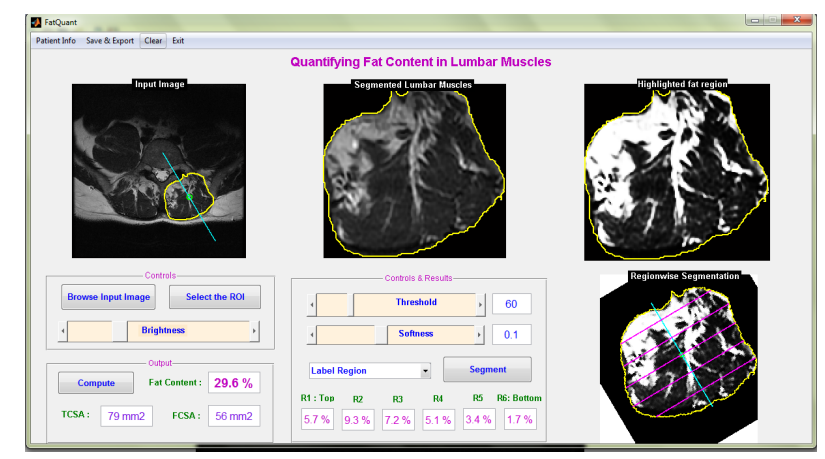

Fig. 10. GUI screen shot: Quantifying fat content in ES Muscles (Right)

\section{ACKNOWLEDGEMENT}

This publication has emanated from research conducted with the financial support of Science Foundation Ireland (SFI) under grant number SFI/12/RC/2289.

\section{REFERENCES}

[1] Per Kjaer, Tom Bendix, Joan S Sorensen, Lars Korsholm, Charlotte Leboeuf-Yde. Are MRI-defined fat infiltration in the multifidus muscles associated with low back pain? BMC medicine, 5(1), 2007.

[2] Adriano Pezolato, Everaldo Encide de Vasconcelos, Helton Luiz Aparecido Defino, Marcello Henrique Nogueira-Barbosa. Fat infiltration in the lumbar multifidus and erector spinae muscles in subjects with sway-back posture. European Spine Journal, 21(11), 2158-2164, 2012.
[3] Roseline D'hooge, Barbara Cagnie, Geert Crombez, Guy Vanderstraeten, Mieke Dolphens, Lieven Danneels. Increased intramuscular fatty infiltration without differences in lumbar muscle cross-sectional area during remission of unilateral recurrent low back pain. Manual therapy, 17(6), 584-588, 2012.

[4] Lucia Ballerini, Anders Hogberg, Gunilla Borgefors, Ann-Christin Bylund, Ann Lindgard, Kerstin Lundstrom, Olivier Rakotonirainy and Bassam Soussi. A segmentation technique to determine fat content in NMR images of beef meat. IEEE Transactions on Nuclear Science, 49(1), 195199, 2002.

[5] Lucia Ballerini, Anders Hogberg, Gunilla Borgefors, Ann-Christin Bylund, Ann Lindgard, Kerstin Lundstrom, Olivier Rakotonirainy and Bassam Soussi. Testing MRI and image analysis techniques for fat quantification in meat science. IEEE Nuclear Science Symposium Conference Record,Vol.3, 18-136, 2000.

[6] Craia A Ranson, Angus F Burnett, Robert Kerslake, Make E Batt, Peter B O'Sullivan. An investigation into the use of MR imaging to determine the functional cross sectional area of lumbar paraspinal muscles. European Spine Journal, 15(6), 764-773, 2006.

[7] Christophe Demoulin, Jean-Michel Crielaard, Marc Vanderthommen Spinal muscle evaluation in healthy individuals and low-back-pain patients: a literature review. Joint Bone Spine, 74(1), 9-13, 2007.

[8] Bernard Mengiardi, Marius R Schimd, Norbert Boos, Christian WA Pfirrmann, Florian Brunner, Achiem Elfering, Juerg Hodler. Fat Content of Lumbar Paraspinal Muscles in Patients with Chronic Low Back Pain and in Asymptomatic Volunteers: Quantification with MR Spectroscopy. Radiology, 240(3), 786-792, 2006.

[9] DF Kader, D Wardlaw, FW Smith. Correlation between the MRI changes in the lumbar multifidus muscles and leg pain. Clinical radiology, 55(2), 145-149, 2000.

[10] Annina Ropponen, Tapio Videman, Michele C Battie. The reliability of paraspinal muscles composition measurements using routine spine MRI and their association with back function. Manual Therapy, 13(4), 349356, 2008.

[11] C Datin, A Colin, J-Y Boire, J M Franconni, I Tauveron, A Veyre, $\mathrm{M}$ Zanca. Determination of fat and water contents in human from S̈AT:-MRI sequences using automatic image segmentation. 18th Annual International Conference of the IEEE Engineering in Medicine and Biology Society, EMBS 1996. Bridging Disciplines for Biomedicine, proceedings, vol.2, 748-749, 1996.

[12] Karen L Barker ,Delva R Shamley, David Jackson. Changes in the crosssectional area of multifidus and psoas in patients with unilateral back pain the relationship to pain and disability. Spine,29(22), E515-E519, 2004.

[13] Kjersti Storheim, Inger Holm, Ragnhild Gunderson, Jens Ivar Bros, Kari Bo. The effect of comprehensive group training on cross-sectional area, density, and strength of paraspinal muscles in patients sick-listed for subacute low back pain. Journal of spinal disorders \& techniques, 16(3), 271-279, 2003.

[14] Paul L Flicker, James L Fleckenstein, Kathryn Ferry, Jerri Payne, Christine Ward, Tom Mayer, Robert W Parkey, Ronald M Peshock. Lumbar muscle usage in chronic low back pain: magnetic resonance image evaluation. Spine, 18(5), 582-586, 1993.

[15] Gabrielle Hultman, Margareta Nordin, Helena Saraste, Hans Ohlsen. Body composition, endurance, strength, cross-sectional area, and density of MM erector spinae in men with and without low back pain. Journal of Spinal Disorders \& Techniques,6(2), 114-123, 1993.

[16] Parkkola R, U Rytokoski, M Kormano. Magnetic resonance imaging of the discs and trunk muscles in patients with chronic low back pain and healthy control subjects. Spine, 18(7), 830-836, 1993.

[17] Sanjum P Samagh, Charles D Rosen, Karimdad Otarodifard, Matthew Kornswiet, Gabe Palmer, Thay Q Lee. New method for determining apparent axial center of rotation of lumbar and thoracic spine segments. Journal of Rehabilitation Research \& Development, 48(5), 2011.

[18] Richard C Schafer. Motion Palpation and Chiropractic Technique. Chapter 5: Lumbar Spine, Motion Palpation Institute, 1989.

[19] David A Lisle. Imaging for Students. Chapter 9: Anatomy of Spine, Hodder Arnold, 2012 